\title{
Revascularização do miocárdio em pacientes após a oitava década de vida
}

\author{
Rui Manuel Sequeira de ALMEIDA *,**, José Dantas LIMA JR. *,**, José Fernando MARTINS**, \\ Danton Richlin Rocha LOURES*
}

RBCCV 44205-579

\begin{abstract}
Almeida RMS, Lima Jr. JD, Martins JF, Loures DRR - Revascularização do miocárdio em pacientes após a oitava década de vida. Rev Bras Cir Cardiovasc 2002; 17(2): 08-14.

RESUMO: Objetivo: Avaliar a morbi-mortalidade hospitalar e a qualidade de vida de pacientes, acima de 70 anos de vida, submetidos à operação de revascularização do miocárdio (CRM).

Casuística e Métodos: No período de julho de 1992 a fevereiro de 2000, foram realizadas 507 CRM, no Serviço de Cirurgia Cardiovascular do Instituto de Moléstias Cardiovasculares de Cascavel. Em 70 destes casos os pacientes tinham idade igual ou superior a 70 anos. Neste grupo predominou o sexo masculino, em $57 \%$ dos casos, e a idade média foi de 72,9 anos (70-85 anos). Vinte e seis pacientes apresentavam hipertensão arterial sistêmica, 25 doença pulmonar obstrutiva crônica importante, 17 diabete melito e 8 insuficiência renal crônica, no pré-operatório. Trinta e sete pacientes apresentavam infarto agudo do miocárdio (IAM) pré-operatório, sete haviam sido submetidos à angioplastia transluminal percutânea, sete apresentavam lesão de tronco de artéria coronária esquerda e um havia sido submetido à CRM anteriormente. Foram realizados 2,8 enxertos/ paciente, sendo usados condutos arteriais em $53 \%$ dos casos. Foi realizada endarterectomia em sete artérias, aneurismectomia de ventrículo esquerdo em sete pacientes e ventriculectomia parcial esquerda em um. A operação foi realizada em caráter de emergência em nove casos. Houve necessidade de contrapulsação aórtica em quatro pacientes.
\end{abstract}

Resultados: O tempo médio de permanência na UTI e no hospital foi de 4 (1-24) e 12,2 (3-34) dias, respectivamente. A mortalidade hospitalar geral foi de 7,1\%. Quando analisada por subgrupos, a mortalidade dos pacientes de 70 a 74 anos (57 casos) foi de 5,3\%, e a dos últimos 35 casos de $2,8 \%$. No pós-operatório imediato, as complicações mais freqüentes foram: insuficiência respiratória (10), arritmia atrial (7), alteração de conduta (6), infecção pulmonar (6), embolia pulmonar (5), síndrome de baixo débito (4), IAM (3), AVC (3), insuficiência renal aguda (4) e mediastinite (1). No seguimento tardio, quatro $(6,1 \%)$ pacientes foram a óbito, dois deles por causas não cardíacas.

Conclusão: Diante destes resultados, os autores acreditam que a CRM pode ser realizada em indivíduos com idade superior a 70 anos, com mortalidade semelhante ao grupo total de pacientes submetidos à operação de revascularização do miocárdio. Entretanto, considerandose as altas taxas de morbidade, há necessidade de indicação criteriosa e de preparo rigoroso para diminuir as complicações pós-operatórias.

DESCRITORES: Coronariopatia, cirurgia. Revascularização miocárdica, mortalidade. Revascularização miocárdica, idoso.

Trabalho realizado no Serviço de Cirurgia Cardiovascular do Instituto de Moléstias Cardiovasculares de Cascavel (IMCC) e na Universidade Estadual do Oeste do Paraná (UNIOESTE). PR, Brasil.

Recebido para publicação em julho de 2001.

* Do Instituto de Moléstias Cardiovasculares de Cascavel.

** Da Universidade Estadual do Oeste do Paraná.

Endereço para correspondência: Rui Manuel Sequeira de Almeida - Instituto de Moléstias Cardiovasculares de Cascavel - Policlínica de Cascavel, R. Maranhão, 790 sala 402. Cascavel, PR, Brasil. CEP: 85806-050. e-mail: almeidar@certto.com.br 


\section{INTRODUÇÃO}

Apesar de haver várias alternativas para o tratamento da doença arterial coronariana (DAC), como o tratamento clínico e a angioplastia transluminal com cateter balão, com ou sem o uso de dispositivos autoexpansíveis endovasculares, a operação de revascularização do miocárdio (CRM) é uma opção com indicações precisas e com bons resultados a médio e longo prazo. O número de pacientes idosos que podem ser candidatos a CRM, por doença aterosclerótica coronariana grave, tem aumentado, em razão do aumento da expectativa de vida da população geral, apesar da operação cardíaca em idosos ser um procedimento de alto risco, em virtude de um grande número de pacientes ser também portador de outras doenças sistêmicas ${ }^{(1,2)}$.

A mortalidade inicial para pacientes idosos submetidos à CRM tem sido relatada como podendo ser até $22 \%$, dependendo da urgência do procedimento(3). Partindo do princípio de que o risco cirúrgico é alto, a pergunta que se faz é se os resultados a médio e longo prazo de CRM são satisfatórios e se há indicação para este tipo de procedimento. Este estudo foi realizado, retrospectivamente, para avaliar a eficácia da CRM, numa série consecutiva de pacientes com idade igual ou superior a 70 anos, avaliando a morbi-mortalidade cirúrgica e a qualidade de vida dos pacientes livres de angina.

\section{CASUÍSTICA E MÉTODOS}

Entre julho de 1992 a fevereiro de 2000, 507 pacientes foram submetidos à CRM, com ou sem o uso de circulação extracorpórea, no Instituto de Moléstias Cardiovasculares de Cascavel (Tabela 1). Destes pacientes, $70(13,8 \%)$ tinham idade superior a 70 anos (média de 72,8 anos) em um intervalo de 70 a 85 anos e $40(57,1 \%)$ eram do sexo masculino. Vinte e três por cento (16/70) dos pacientes estavam em classe funcional III e IV da "New York Heart Association" e 52,9\% apresentavam pelo menos um infarto do miocárdio anteriormente. A parede anterior foi afetada pelo IAM em 18 pacientes, a inferior em 15 e a lateral em 11 casos. A lesão de tronco da artéria coronária esquerda esteve presente em sete (10\%) casos, sendo que 43 pacientes $(61,4 \%)$ apresentaram acometimento de três ou mais vasos arteriais, 24 $(34,3 \%)$ de dois vasos e apenas três $(4,3 \%)$ de uma única artéria. Estes três pacientes uniarteriais foram aceitos para operação por terem sido rejeitados para tratamento pelo grupo da cardiologia intervencionista e persistirem com sintomatologia, apesar do tratamento clínico. Quarenta e oito pacientes (68,6\%) apresentavam doenças associadas, sendo que 26 $(37,1 \%)$ eram portadores de hipertensão arterial sistêmica, $25(35,7 \%)$ de doença pulmonar obstrutiva
TABELA 1. DISTRIBUIÇÃO ANUAL DOS PACIENTES SUBMETIDOS A CRM

\begin{tabular}{|cccc}
\hline Ano & $\begin{array}{c}\text { Número de } \\
\text { CRM }\end{array}$ & $\begin{array}{c}\text { Número de CRM } \\
\text { acima da } \mathbf{8}^{\mathbf{a}} \text { década }\end{array}$ \\
\hline 1992 & 7 & --- & --- \\
\hline 1993 & 32 & 2 & $6,25 \%$ \\
\hline 1994 & 44 & 4 & $9,09 \%$ \\
\hline 1995 & 46 & 3 & $6,52 \%$ \\
\hline 1996 & 81 & 9 & $11,11 \%$ \\
\hline 1997 & 92 & 15 & $16,30 \%$ \\
\hline 1998 & 87 & 9 & $10,34 \%$ \\
\hline 1999 & 105 & 23 & $21,90 \%$ \\
\hline 2000 & 13 & 5 & $38,46 \%$ \\
\hline Total & $\mathbf{5 0 7}$ & $\mathbf{7 0}$ & $\mathbf{1 3 , 8 1 \%}$ \\
\hline
\end{tabular}

crônica, 17 (24,3\%) de diabete melito e oito $(11,4 \%)$ de insuficiência renal crônica. Os pacientes apresentavam como características físicas, peso médio de $71.3 \mathrm{~kg}$ (46 a $137 \mathrm{~kg}$ ), altura média de 1,63m (1,43 a 1,80m), superfície corpórea média de $1,78 \mathrm{~m}^{2}$ (1,41 a 2,45 $\left.\mathrm{m}^{2}\right)$ e índice de massa corpórea médio de 27 (18 a 43) - Tabela 2. Em 23 pacientes a operação não foi eletiva e foi classificada como urgente em 14 (20\%) e como emergência em nove $(12,9 \%)$ dos ca-

TABELA2. DISTRIBUIÇÃO DO GRUPO QUANTO AO ÍNDICE DE MASSA CORPÓREA

\begin{tabular}{ccc}
\hline MASSA CORPÓREA & № & $\%$ \\
$<25$ & 17 & $24,29 \%$ \\
$25-30$ & 39 & $55,71 \%$ \\
$31-35$ & 10 & $14,29 \%$ \\
$36-40$ & 3 & $4,29 \%$ \\
$>40$ & 1 & $1,43 \%$ \\
\hline
\end{tabular}

sos. Pacientes com angina instável e portadores de lesão de tronco e/ou óstio foram considerados casos de urgência, sendo os portadores angina de repouso, insuficiência cardíaca pós-IAM, arritmias ventriculares ou com infarto agudo do miocárdio em evolução, como de emergência. Para avaliação da função ventricular esquerda foi usada a fração de ejeção global (FE), obtida por angiografia digital, sendo considerada 
disfunção grave quando a $\mathrm{FE}$ era $<40 \%$, moderada quando a FE era $>40 \%$ e $<50 \%$ e função normal quando a FE era $>50 \%$ (Tabela 3). Sete pacientes $(10 \%)$ haviam sido submetidos à angioplastia transluminal percutânea, com o uso de dispositivos autoexpansíveis intracoronarianos em $28,6 \%$ dos casos, e um à CRM prévia.

TABELA 3. CORRELAÇÃO DA FUNÇÃO VENTRICULAR ESQUERDA E MORTALIDADE

\begin{tabular}{lcccc}
\hline $\begin{array}{c}\text { Função } \\
\text { Ventricular } \\
\text { esquerda }\end{array}$ & № & $\%$ & \multicolumn{2}{c}{$\begin{array}{c}\text { Mortalidade } \\
\text { no }\end{array}$} \\
$\begin{array}{l}\mathrm{FE}<40 \% \\
\mathrm{FE}>40-<50 \%\end{array}$ & 16 & $22,86 \%$ & - & - \\
$\mathrm{FE}>50 \%$ & 44 & $62,86 \%$ & 5 & $11,36 \%$ \\
\hline
\end{tabular}

Todas as operações, exceto uma, foram realizadas com auxílio de circulação extracorpórea, sendo usado o oxigenador de bolhas até outubro de 1995 e após esta data o oxigenador de membrana. A canulação foi única no átrio direito e na aorta. A proteção miocárdica foi obtida através de uma hipotermia moderada ( 28 a $30^{\circ} \mathrm{C}$ ), uso de cardioplegia cristalóide a $4^{\circ} \mathrm{C}$, e resfriamento tópico do miocárdio. Todas as anastomoses distais foram realizadas em primeiro lugar, com um só pinçamento aórtico, e as anastomoses distais foram realizadas com o coração batendo e pinçamento parcial da aorta.

A média de revascularização por paciente foi de 2,76 enxertos, sendo que a artéria torácica interna esquerda foi usada em $52,9 \%$ dos casos. Procedimentos estiveram associados em $21,3 \%$ dos pacientes, sendo que em sete casos foi realizada a aneurismectomia, linear em seis e a endoventriculoplastia em um (três casos com a retirada de trombos do ventrículo esquerdo), em sete casos a endarterectomia de uma artéria coronária, em um caso a troca da valva mitral por uma prótese de pericárdio bovino (Braile ${ }^{\mathrm{MR}}$ ) e em outro caso uma ventriculectomia parcial. A revascularização miocárdica foi realizada para a parede inferior com 39 enxertos, para a lateral com 54 enxertos e para a anterior com 100 enxertos, num total de 193 enxertos (Tabela 4). Para realizar estas operações foi necessário um tempo médio de circulação extracorpórea de 86,8 (40 a 163) minutos e um tempo médio de pinçamento aórtico de 31,8 (10 a 67) minutos.
TABELA 4. TIPOS DE ENXERTOS E ARTÉRIAS REVASCULARIZADAS

\begin{tabular}{llrl}
\hline \multicolumn{3}{c}{ Artéria Revascularizada } \\
Enxertos Venosos & & 156 \\
DA & 30 & \\
Diagonal & 31 & \\
Diagonalis & 2 & \\
MgCx & 52 & \\
Marginalis & 2 & \\
CD & 34 & \\
& DP & 5 & \\
Enxertos Arteriais & & 37 \\
& DA & 34 & \\
Total & Diagonal & 3 & \\
\hline
\end{tabular}

As complicações pós-operatórias imediatas foram divididas em 3 grupos conforme a sua gravidade, tendo os pacientes, para serem enquadrados num dos grupos, pelo menos uma característica de cada grupo. O grupo I incluía pacientes em que catecolaminas foram utilizadas em baixas doses e por menos de 24 horas, o suporte de ventilação mecânica por períodos menores que 12 horas, não houve aumento dos níveis de creatinina sérica acima de 1,5, houve alteração de conduta, presença de arritmias atriais ou infecção não complicada; o grupo II incluía pacientes em que catecolaminas foram utilizadas em altas doses e por mais de 24 horas, o suporte de ventilação mecânica foi feito por períodos maiores que 12 horas e menores que 48 horas, houve aumento dos níveis de creatinina sem repercussão, houve déficit neurológico transitório, presença de arritmias ventriculares e IAM transoperatório ou infecção complicada; o grupo III incluía pacientes em que catecolaminas foram utilizadas em altas doses, por mais de 24 horas, podendo haver necessidade de balão intra-aórtico, o suporte de ventilação mecânica foi feito por períodos maiores que 48 horas, houve aumento dos níveis de creatinina com oligo-anúria e uso de diálise, houve déficit neurológico com seqüela, presença de fibrilação ventricular ou infecção complicada com sepse.

Em quatro pacientes houve necessidade de suporte circulatório de assistência ventricular esquerda através de balão intra-aórtico (B.I.Ao.), após circulação extracorpórea; em todos os casos foi empregada a técnica descrita por ALMEIDA et al. ${ }^{(4)}$. Em três casos a inserção foi realizada no centro cirúrgico e em um na Unidade de Terapia Intensiva, todos eles por síndrome de baixo débito, refratária ao uso de catecolaminas em altas doses. A mortalidade hospitalar foi definida como óbito que ocorreu nos primei- 
TABELA 5. TIPO DE COMPLICAÇÕES PÓS-OPERATÓRIAS

\begin{tabular}{|c|c|c|c|c|c|c|}
\hline $\begin{array}{c}\text { Tipo de } \\
\text { Complicações }\end{array}$ & Cardíacas & Respiratórias & Neurológicas & Nefrológicas & Infecciosas & Total \\
\hline Grupo I & 7 & - & 4 & - & - & 11 \\
\hline Grupo II & 7 & 13 & 1 & 2 & 1 & 24 \\
\hline Grupo III & 3 & 10 & 3 & 2 & $\ldots$ & 18 \\
\hline Total & 17 & 23 & 8 & 4 & 1 & 53 \\
\hline
\end{tabular}

ros 30 dias pós a CRM ou durante a hospitalização. Todos os pacientes foram seguidos no pós-operatório, sendo contatados por telefone e/ou o seu médico assistente, para determinar sua classe funcional, presença ou ausência de angina e a incidência de novas internações relacionadas ao problema anterior.

\section{RESULTADOS}

A revascularização do miocárdio foi considerada incompleta em 12 pacientes $(17,2 \%)$, definindo-se como revascularização completa toda aquela em que qualquer vaso com lesão acima de $70 \%$ foi revascularizado, independente de seu diâmetro ou da severidade de acometimento da doença no vaso, à exceção do tronco da artéria coronária esquerda onde foi considerado uma lesão obstrutiva de pelo menos $50 \%{ }^{(5)}$. O tempo médio de permanência dos pacientes na UTI foi de 4 (1-24) dias. A alta hospitalar ocorreu em média após 11,9 (3-34) dias de permanência hospitalar, sendo que os pacientes obtiveram alta em média 9,7 dias após a operação. Cinco dos 70 pacientes, com mais de 70 anos foram a óbito no hospital, apresentado mortalidade imediata de $7,1 \%$. Dos cinco óbitos, 3 estavam relacionados a causas cardíacas (IAM transoperatório e arritmia) e outros 2 a não cardíacas (pulmonar e neurológica). A mortalidade imediata para as operações eletivas foi de $2,1 \%$ em contraste com a de 14,2\% para as de urgência e de $22,2 \%$ para as de emergência. Também houve diferenças quando a mortalidade foi analisada por faixa etária, sendo de $5,3 \%$ no grupo de 70 a 74 anos (3/ 57), $11,1 \%$ (1/9) no grupo de 75 a 79 anos e $25 \%$ (1/ 4) no grupo com 80 anos ou mais. Dentre os últimos 35 pacientes operados, incluindo oito casos de urgência e emergência, houve apenas um óbito hospitalar, num caso eletivo, ficando a mortalidade em 2,9\%. Vinte e dois pacientes $(31,4 \%)$ foram extubados pre- cocemente, isto é, o seu tubo endotraqueal foi retirado num período até oito horas após a sua chegada à Unidade de Terapia Intensiva, definição esta usada por outros autores ${ }^{(6)}$. As complicações pós-operatórias estiveram presentes em $35(53,8 \%)$ dos pacientes que receberam alta hospitalar, conforme a Tabela 5. Os 65 pacientes que receberam alta hospitalar foram seguidos por um período de 1 a 70 meses. No momento da alta hospitalar nenhum paciente referiu angina. Houve 5 óbitos tardios (7,7\%), sendo dois casos por causas cardíacas (falência ventricular esquerda e arritmia) e três por não cardíacas (acidente vascular cerebral, pneumopatia e tumor de próstata). A recuperação funcional destes pacientes foi excelente, sendo que $81,6 \%(49 / 60)$ encontram-se na classe funcional I ou II da NYHA e apenas dois pacientes referem angina aos esforços.

\section{COMENTÁRIOS}

Com o envelhecimento da população, os sinais e sintomas da DAC, em pacientes mais idosos, começam a aparecer e aumenta o número de doentes com indicação para revascularização coronariana. Vários trabalhos têm relatado os resultados da CRM em pacientes na oitava década de vida em função deste ser um grupo diferente de pacientes, principalmente em relação às associações com outras doenças no préoperatório e às complicações que podem aparecer após a operação. Nesta casuística este grupo de pacientes representou $13,8 \%$ do total de pacientes submetidos à CRM, o que, apesar do relativo número pequeno da amostra, está em acordo com a experiência de outros autores ${ }^{(3,7-10)}$. A distribuição entre os sexos também foi equivalente e condizente com os estudos anteriores. As características físicas dos pacientes usadas na maioria dos estudos, como altura e peso, em pacientes nesta faixa etária não são 
tão relevantes, apenas o índice de massa corporéa, e este foi semelhante ao relatado por outros autores ${ }^{(8)}$. A associação com outras doenças comuns nesta faixa etária foi observada, com especial ênfase para a hipertensão arterial e doenças pulmonares. A definição prévia de casos de urgência e emergência permite uma melhor estratificação dos grupos de risco. Não foi observado, nesta casuística, um número significativo de pacientes com lesão de tronco de artéria coronária esquerda, em contraste, com os outros relatos em que este tipo de lesão é apresentado numa alta porcentagem de até $38,9 \%^{(11-13)}$. O número de pacientes que apresentaram IAM prévio (52,9\%), bem como o número de artérias coronárias comprometidas $(61,4 \%$ de triarteriais), mostra não só a severidade da doença arterial coronariana nesta faixa etária, mas também que a grande maioria de pacientes com doença menos evolutiva tem sido tratada com revascularização através de angioplastias. Note-se que apenas três pacientes eram uniarteriais, que já tinham sido rejeitados para ATPC, em virtude dos maus resultados com este tipo de lesões. O número de 2,76 enxertos/ paciente, no grupo de estudo, está relacionado tanto ao número de artérias comprometidas, ao número de infartos prévios como ao número de casos que não foram completamente revascularizados $(17,2 \%)$, semelhante aos resultados de outros autores ${ }^{(7)}$, quer seja pelo diâmetro diminuto das artérias coronárias, como pela pequena área irrigada pelas mesmas.

O número de associações e a sua gravidade, tais como aneurismectomia corrigida pela técnica da aneurismorrafia linear (14) ou pelo método da endoventriculoplastia, com exclusão septal ${ }^{(15)}$, bem como a lesão valvar mitral decorrente da DAC, não estiveram relacionados com a morbi-mortalidade deste grupo. Os tempos médios de circulação extracorpórea e pinçamento aórtico são compatíveis com os de vários grupos, quando analisamos o número médio de enxertos ${ }^{(7,13)}$. O tempo médio de permanência hospitalar após a operação também foi condizente, como obtido por outros autores ${ }^{(7,13)}$, sendo que todos os pacientes que permaneceram por mais de 14 dias apresentaram complicações de ordem respiratória e/ou neurológica ${ }^{(16)}$. Apesar de descrita, a síndrome de baixo débito não foi uma das complicações mais importantes deste grupo, não apresentando relação com a mortalidade hospitalar. A análise da mortalidade operatória levando em conta os diversos subgrupos de idade, tipo de operação e a experiência do Serviço, mostra que os grupos com idade igual ou superior a 75 anos não são representativos, pelo pequeno número de casos, porém estão de acordo com a literatura mundial|(3,17). As operações eletivas têm os meIhores resultados, quando comparadas às de urgência e emergência. Quanto à experiência de Serviço, a
TABELA 6. REVISÃO DA LITERATURA SOBRE MORTALIDADE HOSPITALAR

\begin{tabular}{|c|c|c|c|}
\hline Referência / Ano & Idade & N.ㅇ & Mortalidade \\
\hline Meyer et al. ${ }^{(20)} / 1975$ & $>70$ & 95 & $22,1 \%$ \\
\hline Hochberg et al. ${ }^{(12)} / 1982$ & $>70$ & 75 & $12,0 \%$ \\
\hline Janusz et al. ${ }^{(10)} / 1983$ & $>70$ & 67 & $5,9 \%$ \\
\hline Pelletier et al. ${ }^{(21)} / 1984$ & $>70$ & 67 & $4,5 \%$ \\
\hline Pavie et al. ${ }^{(8)} / 1984$ & $>65$ & 255 & $11,1 \%$ \\
\hline Ennabli et al.(11) / 1986 & $>70$ & 102 & $6,9 \%$ \\
\hline Horvath et al. ${ }^{(3)} / 1990$ & $>75$ & 222 & $10,8 \%$ \\
\hline Cane et al. ${ }^{(22)} / 1995$ & $>80$ & 121 & $9,1 \%$ \\
\hline Williams et al. ${ }^{(17)} / 1995$ & $>80$ & 300 & $11,0 \%$ \\
\hline Mukai et al. ${ }^{(19)} / 1997$ & $>80$ & 11 & $27,0 \%$ \\
\hline Silva et al. ${ }^{(7)} / 1997$ & $>70$ & 41 & $9,9 \%$ \\
\hline Iglézias et al. ${ }^{(8)} / 1997$ & $>70$ & 361 & $9,1 \%$ \\
\hline Deinniger et al. ${ }^{(13)} / 1999$ & $>70$ & 144 & $5,5 \%$ \\
\hline Almeida et al. ${ }^{(*)} / 2002$ & $>70$ & 70 & $7,1 \%$ \\
\hline
\end{tabular}

$\left({ }^{*}\right)$ Presente estudo

diminuição de mortalidade dos primeiros 35 casos $(11,4 \%)$ para os últimos $(2,9 \%)$ mostra a tendência de obtenção de resultados iguais aos demais Centros de Cirurgia Cardiovascular (Tabela 6). Na evolução a médio e longo prazo foi possível observar uma sensível melhora na qualidade de vida dos sobreviventes, sendo que no seguimento observou-se que a grande maioria está livre de angina e sem internações por causa cardíaca, assim como outros autores observaram nos pacientes operados acima de 70 anos de idade $^{(18)}$.

\section{CONCLUSÕES}

Em face dos resultados alcançados, concluise que a CRM neste grupo de pacientes é possível, com uma baixa mortalidade hospitalar, nos casos eletivos, e que os índices de morbidade elevados deveram-se principalmente, a complicações pulmonares. Com a finalidade de diminuir a morbidade pós-operatória, estes pacientes devem ser preparados no período pré-operatório, reduzindo suas repercussões sistêmicas, a fim de ressaltar o controle dos fatores de risco, objetivando diminuir sua influência nas complicações. 
RBCCV 44205-579

Almeida RMS, Lima Jr. JD, Martins JF, Loures DRR - Myocardial revascularization in patients above the eightiest decade of life. Rev Bras Cir Cardiovasc 2002; 17(2): 07-13.

ABSTRACT: Objective: In this study, the hospital morbidity and mortality, in patients older than 70 years of age, submitted to coronary artery bypass grafting (CABG), and in their quality of life.

Material and Methods: Between July 1992 and February 2000, 507 CABG were performed at the Cardiovascular Surgery Department of the "Instituto de Moléstias Cardiovasculares de Cascavel", being 70 patients older than 70 years of age. Fifty seven percent of the patients were male, and the mean age was 72.9 years (70-85 years). Twenty six patients had, pre-operatively, systemic arterial hypertension, 25 severe chronic obstructive pulmonary disease, 17 diabetes mellitus and eight chronic renal insufficiency; thirty seven patients presented with pre-operative myocardial acute infarction, seven had been submitted to a percutaneous transluminal coronary angioplasty, seven had left main coronary disease and one had previous CABG. The mean number of grafts/ patient was 2.8 , being in $53 \%$ arterial conduits. In seven patients an endarterectomy was performed, in another seven a left ventricular aneurismectomy and in one a partial left ventriculectomy. Nine cases were performed in an emergency way. Intraaortic balloon pump was used in four cases.

Results: The mean intensive care and hospital stay was 4 (1-24) and 12.2 (3-34) days, respectively. The global hospital mortality was $7.1 \%$. When analysed by subgroups, the 70 to 74 years of age patients' mortality was $5.3 \%$, and that of the last 35 patients was $2.8 \%$. During the postoperative period the most important complications were respiratory failure (10), atrial arrhythmia (7), mental confusion (6), pulmonary infection (6), pulmonary emboli (5), low output syndrome (4), hospital acute myocardial infarction (3), stroke (3), acute renal insufficiency (4) and mediastinitis (1). In the late follow-up four (6.1\%) patients died, being two cases by non-cardiac causes.

Conclusion: In view of these results, the authors believe that CABG can be performed in patients older than 70 years of age, with a mortality almost as the same as the all group, but due to the high morbidity, we should have strict criteria and preparation, in order to lower the postoperative complications.

DESCRIPTORS: Coronary disease, surgery. Myocardial revascularization, mortality. Myocardial revascularization, aged.

\section{REFERÊNCIAS BIBLIOGRÁFICAS}

1. Rabelo RC, Bernardes RC, Reis Filho FAR, Rabelo W, Marino RL - Revascularização do miocárdio no idoso. Arq Bras Cardiol 1993; 61 (Supl. 3): 182.

2. Iskandrian AS \& Segal BL - Should cardiac surgery be performed in octogenarians? J Am Coll Cardiol 1991; 18: 36-7.

3. Horvarth KA, DiSesa VJ, Peigh PS, Couper GS, Collins Jr. JJ, Cohn LH - Favorable results of coronary artery bypass grafting in patients older than 75 years. J Thorac Cardiovasc Surg 1990; 99: 92-6.
4. Almeida RMSSA, Ferreira MJA, Ribeiro EJ, Loures DRR - Implante cirúrgico do balão intra-aórtico: uso de uma nova técnica. Rev Bras Cir Cardiovasc 1996; 11: 216-9.

5. CASS - Coronary Artery Surgery Study - A randomized trial of coronary artery bypass surgery. survival data. Circulation 1983; 68: 939-50.

6. Higgins $T L-$ Pro: early endotracheal extubation is preferable to late extubation in patients following coronary artery surgery. J Cardiothorac Vasc Anesth 1992; 6: 488-93. 
7. Silva LHF, Nascimento CS, Viotti Jr. LAP Revascularização do miocárdio em idosos. Rev Bras Cir Cardiovasc 1997; 12: 132-40.

8. Iglézias JCR, Oliveira Jr JL, Fels KW et al. Fatores prognósticos na revascularização do miocárdio em pacientes idosos. Rev Bras Cir Cardiovasc 1997; 12: 325-34.

9. Pavie A, Fontanel M, Gandjbakhch I et al. - Openheart surgery in patients over 65 years of age: lesions encountered and immediate postoperative mortality. Ann Cardiol Angeiol (Paris) 1984; 33: 367-72.

10. Janusz MT, Jamieson WR, Causton N, Burr L H, Allen P, Munro A I - Coronary artery bypass in patients over 65 years of age. Can J Surg 1983; 26: 186-8.

11. Ennabli K\& Pelletier LC - Morbidity and mortality of coronary artery surgery after the age of 70 years. Ann Thorac Surg 1986; 42: 197-200.

12. Hochberg MS, Levine FH, Daggett WM, Akins CW, Austen WG, Buckley MJ - Isolated coronary artery bypass grafting in patients seventy years of age and older: early and late results. J Thorac Cardiovasc Surg 1982; 84: 219-23.

13. Deininger MO, Oliveira OG, Guedes MGA et al. - Cirurgia de revascularização do miocárdio no idoso: estudo descritivo de 144 casos. Rev Bras Cir Cardiovasc 1999; 14: 88-97.

14. Almeida RMSSA, Lima Jr. JD, Bastos LC, Flores $E Q$, Loures DR - Surgery for correction of anterior left ventricular aneurysm. Congresso Virtual de Cardiologia. http://pcvc.sminter.com.ar/cvirtual/tlibres/ nn2342/tnn2342.htm\#disc
15. Almeida RMS, Lima Jr. JD, Bastos LC, CarvaIho CT, Loures DR - Remodelamento do ventrículo esquerdo pela técnica da endoventriculoplastia com exclusão septal: experiência inicial. Rev Bras Cir Cardiovasc 2000; 15:302-7.

16. Acinapura A J, Rose DM, Cunninghan Jr. N, Jacobowitz I J, Kramer MD, Zisbrod Z - Coronary artery bypass in septuagenarians: analysis of mortality and morbidity. Circulation 1988; 78(3 pt 2): I179-84.

17. Williams D B, Carrillo RG, Traad E A, Wyatt $\mathrm{CH}$, Grahowksi R, Wittels SH, Ebra G - Determinants of operative mortality in octogenarians undergoing coronary bypass. Ann Thorac Surg 1995; 60: 1038-43.

18. Bhattacharya SK, Teskey JM, Cohen M, Kim SW, Barwinsky J - Risks and benefits of open-heart surgery in patients 70 years of age and older. Can $J$ Surg 1984; 27: 150-2.

19. Mukai S, Murata H, Ueda T, Kita K, Lee S Coronary artery bypass grafting in patients aged 80 years or older. Kyobu Geka 1997; 50(8 Suppl): 653-5.

20. Meyer J, Wukasch DC, Seybold-epting W et al. - Coronary artery bypass in patients over 70 years of age: indications and results. Am J Cardiol 1975; 36: 342-5.

21.Pelletier LC \& Castonguay $Y$ - Open-heart surgery after 70 years of age. Results in 136 patients. Ann Cardiol Angeiol (Paris) 1984; 33: 373-8.

22. Cane ME, Chen C, Bailey BM et al. - CABG in octogenarians: early and late events and actuarial survival in comparison with a matched population. Ann Thorac Surg 1995; 60: 1033-7. 